\title{
Study on Thinking Evolution based ant Colony Algorithm in Typical Production Scheduling Application
}

\author{
Xianmin $\mathrm{Wei}^{1}$ and Peng Zhang ${ }^{2}$ \\ ${ }^{1,2}$ School of Computer Engineering, Weifang University \\ 5147 Eastern Dongfeng Street, Weifang 261061, China \\ wfxyweixm@126.com
}

\begin{abstract}
Aiming at solving the NP-hard workshop production scheduling problems, proposed one kind based on mind evolutionary algorithm. The algorithm in the traditional ant colony algorithm is established, and the combination of evolutionary thought and local optimization idea overcomes the basic ant colony algorithm is easy to fall into local optimal defects, the improved state transition rules, defining a pheromone range, improve the pheromone update strategy, and the increase of neighborhood search. Experimental results show that, for a typical production scheduling problems, based on mind evolutionary ant colony algorithm can obtain the optimal solution in theory, optimal solution, the solution and average three indicators are better than the basic ant colony algorithm, showed good performance.
\end{abstract}

Keywords: Evolutionary, Ant colony algorithm, Production scheduling, local search

\section{Introduction}

At present, with the formation of the markets globalization competition among manufacturing enterprises has been increasingly fierce. Competition makes a variety of product life cycles are getting shorter, the number of product varieties rapidly expands, a lot of small species production model the past has been changed, but are gradually becoming more varieties of small batch production. Under such changes, produced varieties are changing at each day, even scheduling program arranged due to a sudden change from the customer, also needs to change. Machine tools and equipment damage may occur at any sudden situations, make the production and processing environment continues changing. Facing with these changes, to maintain the company's production capacity and efficiency at a high level has always, how we can solve the enterprise for production management in this issue has become the focus of attention problem of the current academic and specific enterprises are [1].

Production planning and scheduling are the most critical component and most core in process of production and management, its management effects has a direct and significant impact on the production performance of a company, with a more accurate peripheral information, if coupled with effective production planning and scheduling, to maximize production and business efficiency for the company is a very important functional role, also it is more able to meet customers' demands [2].

Research on the production scheduling problem stems from the 1950s, this is a crosscutting areas of multiple professional disciplines, which involving professional fields including mathematics, industrial engineering, computer engineering, control engineering, logistics and other disciplines knowledge and skills [3]. For an enterprise, if it can be reasonably effective scheduling and dispatch process can maximize the efficiency of production management of the trial production equipment, to avoid vacant equipment to get rid of bottlenecks in the production process to a large extent, to achieve speeding up 
the production process, and to less inventory backlog, can greatly reduce the production and operation costs, and to increase profitable probability and proportion [4]. Production scheduling problem has always been a theoretical problem not well addressed, the study of the production scheduling problem, has attracted great attention of relevant experts and scholars on this issue, many people with varying degrees of research have made some achievements [5].

However, most workshop production scheduling is more difficult to solve, which are NP-HARD problems that are simple to describe, but if you want to seek an answer to the problem, then it is not so easy. Even considered asking for a correct answer is more difficult [6]. The ways we seek answers to NP-HARD problem are often used with the current heuristic algorithms or random search algorithms, such as particle swarm optimization, ant algorithms and genetic algorithms, etc. [7]. To this end, we carried out related research and exploration for production scheduling problems, together to study the problem of these projections processing, we can propose a new calculation method to solve the problem on the basis of these previous algorithms, which can provide important reference value [8] to solve the problem of a path similar to other related fields. Thus, we can know, research and production scheduling problem has great practical significance, also it has considerable theoretical significance.

This paper is mainly for solving NP-hard problems in the workshop production scheduling through in-depth study of the nature of the problem, designs the ant colony algorithm for solving approach, and based on the ant colony algorithm, combines with evolutionary thinking and local optimization, proposed ant colony algorithm based on the evolution of the thinking process, the calculation method for the calculation process was re-designed, also the process of solving is according to the characteristics of different types.

\section{Basic Principles of an Ant Colony Algorithm}

The so-called ant colony algorithm is a kind of bionic calculation method from the living world, and can be called ant algorithm inspired by the study of the nature reality and the behavior of ants [9]. This kind of calculation is universal, random, stepped from the behavioral characteristics of nature ants, humans absorb the wisdom of other creatures in nature, or after human nature active sentiment to apply these methods from other creatures in nature to the problem of human society in the past, in order to solve problems encountered in human societies, it is the wisdom of universal human development and people's thinking has been greatly enhance the level of results. The ant colony algorithm absorbs characteristics of the behavior of ants, using its special internal search mechanism to make obvious application results in a series of difficulties for the portfolio optimization and solving problems, we will call it ant system, simulation concept of artificial ants were used in solving the problem.

First, the ant has a special mechanism for effective search of food, which provides references of ideas for mankind to solve many difficult problems encountered during the production life [10]. It summed up the sequential rules in the process of ants looking for food. Track intensity is $\tau_{i j}$ of path $(i, j)$, the number of ants, ant the unit track pheromone leaving by ant $k$ is $\Delta \tau_{i j}^{k}$, the transition probability is $p_{i j}^{k}$ of ant $k$, and the importance of the unit track leaving is $\alpha(\alpha \geq 0)$, the importance of its path showing is $\beta(\beta \geq 0)$, the degree of residual pheromone is $\rho(0 \leq \rho<1)$.

$\tau_{i j} 、 \Delta \tau_{i j}^{k}$ and $p_{i j}^{k}$ have different forms of expression can be expressed according to the specific problem. In general, the transition probability of ant $k$ : 


$$
p_{i j}^{k}=\left\{\begin{array}{cc}
\frac{\left[\tau_{i j}\right]^{\alpha}\left[\eta_{i j}\right]^{\beta}}{\sum_{u \in \text { allowed }_{k}}\left[\tau_{i j}\right]^{\alpha}\left[\eta_{i u}\right]^{\beta}} & \text { if } \\
0 & \text { otherwise }
\end{array}\right.
$$

Where allowed $_{k}=\{0,1, \cdots, \mathrm{n}-1\}-$ tabu $_{k}$ represents a collection of the next choice of ant $k$. Respectively, $\alpha$ and $\beta$ express accumulated information and the impact of during the movement of ants heuristic factor ants decisions.

The updating equation for the trajectory of strength is:

$$
\tau_{i j}^{\text {new }}=\rho \cdot \tau_{i j}^{\text {old }}+\Delta \tau_{i j}
$$

Where, $\Delta \tau_{i j}=\sum_{k=1}^{m} \Delta \tau_{i j}^{k}$, the number of pheromones track units of ant $k$ is $\Delta \tau_{i j}^{k}$, M.

Dorigo has given three different models:

(1)Ant-Cycle Model

$$
\Delta \tau_{i j}^{k}=\left\{\begin{array}{l}
\frac{Q}{L_{k}}, \text { if }(i, j) \text { on optimal path } \\
0, \text { else }
\end{array}\right.
$$

$\mathrm{L}_{\mathrm{k}}$ is objective function.

(2)Ant-Density Model

(3)Ant-Quantity Model

$$
\Delta \tau_{i j}^{k}=\left\{\begin{array}{l}
Q, \text { if }(i, j) \text { on optimal path } \\
0, \text { else }
\end{array}\right.
$$

$$
\Delta \tau_{i j}^{k}=\left\{\begin{array}{l}
\frac{Q}{d_{i j}}, \text { if }(i, j) \text { on optimal path } \\
0, \text { else }
\end{array}\right.
$$

Where, constant $Q$ represents the intensity number of tracks leaving by ants.

Simple process of ant colony algorithm shown in Figure 1.

Step 1: Initializing Ant (t), i.e., initializing ant colony;

Step 2: Evaluation Ant (t), i.e., calculating $\Delta \tau_{i j}^{k}$ for each ant based on objective function;

Step 3: Placing the pheromone, that in accordance with $\Delta \tau_{i j}^{k}$, pheromone proportionately placed on the path the ants passed (the higher $\Delta \tau_{i j}^{k}$, the pheromone is placed more);

Step 4: ants moving, ants selecting path according to pheromone left by previous ants and their own judgment $p_{i j}^{k}$;

Step 5: pheromone dissipating, pheromones will continue to decay over time. 


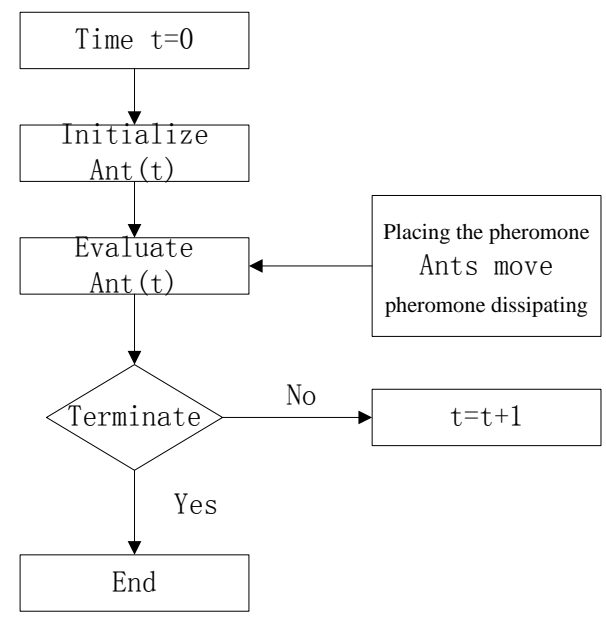

Figure 1. The Simple Process of the Ant Colony Algorithm

\section{Ant Colony Algorithm Based On Evolutionary Thinking}

\subsection{Mind Evolutionary Algorithm}

This algorithm is a simulation of the evolution process of human thinking, in accordance with the views of the algorithm, which considers the human mind, is a process of directed evolution to improve. Mind evolutionary algorithm also proposed these two concepts of alienation and convergence, using a bulletin board approach to fully utilize the evolution of the information, to a larger extent the selectivity of the evolutionary direction has greatly increased, its frame generally shown in Figure 2.

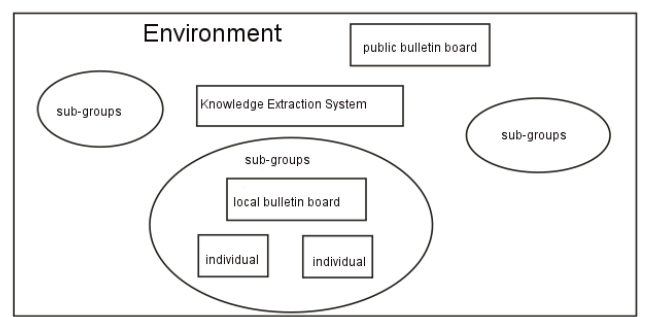

Figure 2. Basic Structural Framework Mind Evolutionary Algorithm

The composition objects are winning subgroups, temporary subgroups, and global bulletin board. In which each of the winning sub-groups and temporary sub-groups are a combination of several indiviiduals and from local bulletin board, each particular individual is assigned a score value according to certain rules, the value is the main frame of reference of environmental optimization.

The two operators of convergence and alienation completed the process of thinking evolution. According to the current understanding, the so-called convergence is by taking the current thinking of others or thinking models to resolve the existing problems, which is an important processes of sub-group individuals in accordance with relevant local information bulletin board competition learning and gradually converge to the local optimal solution. The so-called alienation, that is, when people solve important problems, not through more conventional ways of thinking modes, but getting rid of the conventional thinking, according to new ways of thinking to think and analyze and solve problems, which is a new way to solve the problem, this is a seeking global optimal solution process under the guide of the global bulletin board to achieve. 
When conducting thinking during evolution of the algorithm, we must first set the parameters, and initialize local and global bulletin board, after a number of individuals randomly generated, the winning sub-groups and the temporary sub- groups are identified as the initial group. Then, based on the designed strategy and approaches to conduct alienation operation and convergence operation in accordance with the conditions for the termination, at this time we can solve the problem.

\subsection{Ant Colony Algorithm based on Evolutionary Thinking}

Ant colony algorithm based on evolutionary thinking, after the ant colony algorithm, internal sub-groups carried out the convergence operation, and then its associated pheromone was recorded in the local bulletin board. Ant colony algorithm fully utilized convergence and alienation operation in the thinking process of evolution, it can try to jump out of local optimum, and the search space has been a greater degree of improvement. Integral parts of the designed algorithm proposed in this paper are:

First, individuals and sub-groups. In this calculation method, taking the solution of the problem as an individual, while the sub-groups have the two categories of winning subgroups and the provisional sub-groups.

Second, the bulletin board. Sub-group individual posted with the local bulletin board a, it can be between sub-groups and an individual provides an opportunity for information exchange.

Specific program:

First, initialize. For the first time, setting the number of sub-groups $N_{s}+N_{t}$ as its initial group, and we have considered each group as ant colony system, after which each parameter is initialized, the initial pheromone distribution of various groups conducted to determine, each real estate groups in the solution space give birth to a random number of $M$ individuals, and based on the resulting scores to determine $N_{s}$ winning aim $N_{t}$ temporary sub-groups, after that, update the local bulletin board.

Second, the convergence operation performed. To carry out the evolutionary process inside the various groups through ant colony algorithm, in accordance with its ant colony algorithm for each search, then each individual score is calculated, after looking for the best individual to be the winner, update local bulletin board pheromone distribution of information, winner can provide learning for the ants on local bulletin boards, then we repeat the search process, during the search of certain of $\mathrm{N}$ times when it is still difficult to find a more excellent individuals, we believe that this group has been mature, and the resulting scores of winner is the final score of this mature group.

Third, alienated operation. After a winning sub-group was mature, we have to carry out local search, if their score is improved, then the pheromone distribution as well as individual information on the bulletin board is updated, else give up the winning subgroups. For temporary sub-groups winning sub-groups to give up, the bulletin board pheromone distribution was reserved, and continued to re-generate new groups after that.

Fourth, repeat the alienation and convergence operations, in accordance with the designed conditions for solving the problem.

The algorithm flowchart shown in Figure 3. 


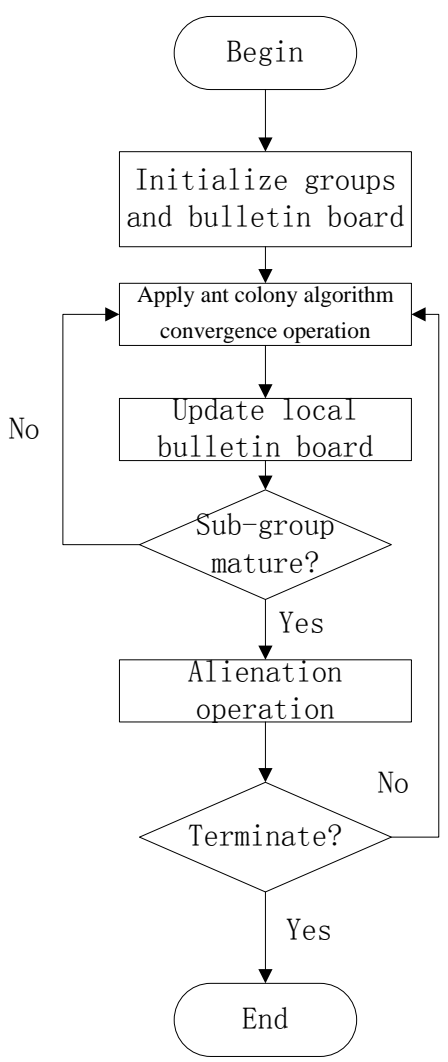

Figure 3. Ant Colony Algorithm Flowchart

\section{Ant Colony Algorithm of Typical Production Scheduling Problem}

\subsection{Typical Job Shop Problem}

Job shop problems study that $\mathrm{n}$ work-piece on $\mathrm{m}$ machines for processing, processing time and sequence is known, and the purpose of our scheduling is that for all the machines and technical constraints of compatibility, how to determine processing order on the work-piece, so as to achieve the purpose of optimizing the processing performance indicators.

And with the following constraints:

Firstly, no precedence constraints between different parts of the supply and demand.

Secondly, during processing a procedure break not allowed.

Finally, the same job cannot be processed at the same time on two machines.

For the actual production of Job shop, and the following aspects of the ant colony algorithm was improved.

\subsection{Improved State Transition Rules}

When ants select the next target in accordance with the following strategies:

$$
j=\left\{\begin{array}{l}
\text { according to } E Q(1), q \leq q_{0} \\
h, q\rangle q_{0}
\end{array}\right.
$$

Among them, the processing time $T_{i j}$ of the $i$ work-piece on the $j$ machine was selected as ants selection heuristic information, that is $\eta=1 / T_{i j}$. 
$h$ was the randomly selected target outside set $t a b u_{k}, q_{0}$ was for a given parameter, $0<q_{0}<1, q$ is a uniformly distributed random number between $(0,1)$, which generated each time when select the target.

This increases the likelihood for target with smaller pheromone values is selected, and jumps out of a local solution. However, if $q_{0}$ is larger, then the possibility of $q \leq q_{0}$ is large, most ants according to Eq.(1) to select the next target, influenced by pheromones relatively large, so there will be the situation for a lot of ants search the same path, which is not conducive to seek optimum solution. If $q_{0}$ is small, then the probability of $q>q_{0}$ increases, when the ants select the next target without been affected by the size of the pheromone, expand the search solution space, but the search showed randomness and not easy to converge.

In order to prevent the search into a local optimal solution, to easily find the global optimal solution, at the beginning of the algorithm $q_{0}$ is greater value, on middle-way $q_{0}$ is small value in order to expand the search space, the latter $q_{0}$ then takes a larger value, accelerating convergence of the algorithm. We take some strategic value of $q_{0}$ and the number ${ }^{n}$ of iterations, the relationship between the two is the following:

$$
q_{0}= \begin{cases}c_{1} & \left(0<n<n_{1}\right) \\ c_{0} & \left(n_{1}<n<n_{2}\right) \\ c_{1} & \left(n_{2}<n<N\right)\end{cases}
$$

Wherein $N$ is the maximum number of iterations, under normal circumstances, $0.8<c_{1}<1,0<c_{0}<0.2$, the when the iteration number of the algorithm is $n_{1}$ and the iteration number of the algorithm is $n_{2}, q_{0}$ values are altered according to Eq. (7).

\subsection{Defining the Scope of Pheromone}

During the process of conducting path optimization, in order to avoid stagnation and spread for ant colony algorithm, on each path possible pheromone concentration will be limited between $\left[\tau_{\min }, \tau_{\max }\right], \tau_{\min }$ can make each step in the ant basic options strategy has the possibility of being selected, and $\tau_{\max }$ can avoid the pheromone on path is much larger than any other path, so that all the ants are concentrated up to the same path. Values outside this range will be forced to set to $\tau_{\text {max }}$ or $\tau_{\text {min }}$, to ensure understanding of the search space.

\subsection{Pheromone Update Strategy}

If the elite policy record was used, and only reward for history optimal solution, ant colony algorithm can be further enhanced positive feedback, and can significantly accelerate the convergence speed. In this paper, two-way convergence strategy was proposed, which makes awards for the history optimal solution, the history worst solution currently can been seen as unacceptable solution and punished, so other ants can be guided as far away from the history worst solution, abandon the opportunity for the worst solutions to be combined into other parts of the composition of the solution, thereby accelerating convergence of the algorithm.

\subsection{Increasing in Neighborhood Search}

In the ant colony algorithm, in order to improve the quality of the solution, the traditional local search algorithms were considered for solving TSP problems, such as 2opt, 3-opt and etc., algorithms were modified to apply to Job shop problem. Here, combined method was considered with neighborhood search algorithm.

Neighborhood structure was usually used as follows:

First, switching. Exchange operation on the position $i$ and $i+1,(i=1,2, \ldots, n-1)$. 
Second, interchangeable. And interchange the operation of the process of $i$ and $j$, where $i \neq j$.

Third, insert. Delete operation $i$, and then insert the operation at the position $j$.

Insertion neighborhood structure is built on the basis of the solution $\pi$ inserted move collect, the definition of insert mobile solution is: Let $v=(i, j)$, which is a pair of position number of solution $\pi$, after mobile insert of a new solution $\pi^{\prime}$ from extraction operations at position $i$, job $v(i)$ was inserted to get access to location $j$. If $i<j$, then:

$\pi^{\prime}=(\pi(1), \ldots, \pi(i-1), \pi(i+1), \ldots, \pi(j), \pi(i), \pi(j+1), \ldots, \pi(n))$,if $i>j$, then: $\pi^{\prime}=$ $(\pi(1), \ldots, \pi(j-1), \pi(i), \pi(j), \ldots, \pi(i-1), \pi(i+1), \ldots, \pi(n))$.

There were a total solution of $(n-1)^{2}$ in neighbor (job $n$ is job number), which can meet the nature of the communication, that is, for any initial solution, after a finite number of iterations it will be able to search the optimal solution.

\section{Simulation Testing and Analysis}

To evaluate the performance of this algorithm, on Windows XP Visual C 6.0 software was applied for simulation, simulation program conducted in their algorithms, including this ant colony algorithm, basic ant colony algorithm for classics FT classes and LA class part of Job-shop scheduling problems are carried out for calculation.

6 FT work-piece of FT class were selected, 6 machines for FT06 Question were simulate objects. Some of the key algorithm parameters are as shown in Table 1.

Table 1. The Algorithm Key Parameters Set

\begin{tabular}{cccc}
\hline Parameters & Value & Parameter & Value \\
\hline $\begin{array}{c}\text { Winning sub-groups } \\
\text { number }\end{array}$ & 1 & $\begin{array}{c}\text { residual factor } \\
\text { pheromone }\end{array}$ & 0.9 \\
\hline $\begin{array}{c}\text { Provisional sub- } \\
\text { groups number }\end{array}$ & 4 & strength of tracks & 100 \\
\hline $\begin{array}{c}\text { Individual number in } \\
\text { sub-groups }\end{array}$ & 30 & $\begin{array}{c}\text { importance of } \\
\text { pheromone }\end{array}$ & 1 \\
\hline $\begin{array}{c}\text { winners number in } \\
\text { global bulletin-board }\end{array}$ & 10 & $\begin{array}{c}\text { importance of heuristic } \\
\text { information }\end{array}$ & 4 \\
\hline $\begin{array}{c}\text { Sub-groups mature } \\
\text { generation }\end{array}$ & 10 & $\begin{array}{c}\text { termination generation of } \\
\text { evolution }\end{array}$ & 50 \\
\hline
\end{tabular}

Randomly run 20 times on the issue, get the FT06 problem by simulating the optimal solution of 55, the results of such statistics were in Table 2.

Table 2. FT06 Problem Simulation Statistical Comparison

\begin{tabular}{cccc}
\hline Algorithm & optimal & worst & average \\
\hline ACA & 73 & 85 & 78.8 \\
ME-ACA & 55 & 58 & 56.2 \\
\hline
\end{tabular}

As can be seen from the above data, for the FT06 problem, ant colony algorithm (MEACA) based on the theory of evolutionary thinking obtained optimal solution, in the optimal solution, the worst solution, and the solution of the three indicators are better than average basic ant swarm, reflecting the better performance.

\section{Conclusions}

Job shop problem is the most common type of scheduling problems among the particular process of enterprises production and operation, in the scheduling process of 
enterprise, full attention must be considered for the impact of processing technology of work-piece on enterprise, meanwhile must also consider personnel configuration, quantity and quality of processing machines and other enterprises in terms of resources and processing of specific practical situations, so finding the optimal solution is a difficult thing. This paper used ant colony algorithm based on evolutionary thinking for typical Job shop problem solving with good effect, for further study on more complicated Job shop a good foundation conditions has been laid.

\section{Acknowledgements}

This work is partly supported by National Natural Science Foundation of China (No. 61471269), Shandong Spark Program (2012XH06005), Weifang municipal Science and Technology Development Program (201301050) and the doctoral scientific research foundation of Weifang University (2104BS12).

\section{References}

[1] S. A. Graves, "Review of production scheduling", Operations Research, vol. 29, (1981).

[2] S. M. Johnson, "Optimal two-and-three-stage production scheduling with set-up times included", Navel Research Logistics Quarterly, vol. 1, (1954).

[3] W. Wan-Liang and W. Qidi, "New method for job-shop scheduling problem Hopfield neural networkbased solution", Computer Integrated Manufacturing Systems-CIMS, vol. 7, no. 12, (2001).

[4] L. Qun, "Flow shop job sorting Improved NEH algorithm", Systems Engineering Theory Methods, vol. 8, no. 4, (1999).

[5] L. Yi, Y. Chun-Ming and S. Yun-Hong, "Improved particle swarm algorithm for Job shop scheduling problem", Systems Engineering and Electronics, vol. 28, no. 4, (2006).

[6] Z. Hong, C. M. Feng and F. Yuncheng, "Solving Flow Shop Scheduling Problem of a genetic algorithm", Beijing University of Aeronautics and Astronautics Journal, vol. 23, no. 4, (1997).

[7] C. Xiong, Y. Fengxia and W. Qidi, "Adaptive simulated annealing Flow-shop scheduling algorithm", Control Theory and Applications, vol. 20, no. 3, (2003).

[8] T. Zhi-Yang, "Artificial immune algorithm Flow shop Problem", Computer Engineering and Applications, vol. 14, (2002).

[9] L. Xu, L. Yuxia and H. Ming, "Fuzzy replacement delivery Tabu Search Algorithm Flow shop scheduling", Dalian Railway Institute, vol. 26, no. 2, (2005).

[10] N. Xin-Zheng, S. Kun and L. Gang, "New research progress and prospects of ant colony algorithm", Computer Applications Research, vol. 24, no. 4, (2007).
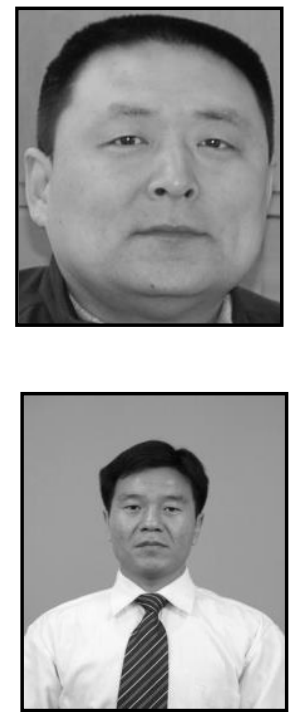

\section{Authors}

Xianmin Wei, He received the M. Sc. degree in computer applications from Shandong Science and Technology University (2005). He is currently an associate professor in school of computer engineering at Weifang University, China. He has published over 30 papers and 3 books in professional fields. Since 2011, he has been a member of IEEE-CS, ACM and CCCF, respectively. His fields of research are focused on swarm intelligent, intelligent sensor networks.

Peng Zhang, He received his $\mathrm{PhD}$ degree of communication and information systems from Shandong University, Jinan, P. R. China, in December 2012. Currently, he is a lecturer in the School of Computer Engineering, Weifang University, Shandong, P. R. China. His current research interests focus on advanced coded modulation, massive MIMO, spatial modulation, cross-layer design, cooperative communications and $4 \mathrm{G} / \mathrm{B} 4 \mathrm{G}$ wireless communications. 
International Journal of Hybrid Information Technology Vol.8, No.6 (2015) 